\title{
A general Schwarz lemma for almost-Hermitian manifolds
}

\author{
VAlentino Tosatti
}

\begin{abstract}
We prove a version of Yau's Schwarz lemma for general almostcomplex manifolds equipped with almost-Hermitian metrics. This requires an extension to this setting of the Laplacian comparison theorem. As an application, we show that the product of two almost-complex manifolds does not admit any complete almostHermitian metric with bisectional curvature bounded between two negative constants that satisfies some additional assumptions.
\end{abstract}

\section{Introduction}

The classical Schwarz-Pick lemma says that a holomorphic map from the unit disc in the complex plane into itself decreases the Poincaré metric. This was later extended by Ahlfors [1] to maps from the disc into a hyperbolic Riemann surface, and by Chern [5] and $\mathrm{Lu}$ [18] to allow more general domains and targets. A major advance was Yau's Schwarz lemma [28], which says that a holomorphic map from a complete Kähler manifold with Ricci curvature bounded below into a Hermitian manifold with holomorphic bisectional curvature bounded above by a negative constant is distance decreasing up to a constant depending only on these bounds. This proved to be extremely useful in differential geometry and complex analysis (see, for example, [17]). Later generalizations of this result were mainly in two directions: relaxing the curvature hypothesis or the Kähler assumption (see $[3,11,20])$ or proving similar results for harmonic maps of Riemannian manifolds [10].

Here we take a different direction and generalize Yau's Schwarz lemma to the case when the complex structures are not integrable. Recently, there has been a lot of interest on geometric and analytic aspects of almostcomplex manifolds (see $[13,24]$ ), also in relation with symplectic geometry

Part of this work was carried out while the author was visiting the Mathematics Department of UCLA and the Morningside Center of Mathematics in Beijing; the author is supported in part by a Jean de Valpine Fellowship. 
(see [7,25]) and complex analysis [6]. Our setting is as follows: suppose we are given two almost-complex manifolds $M$ and $\tilde{M}$, equipped with Riemannian metrics compatible with the almost complex structures (we call such data an almost-Hermitian manifold). A map from $M$ to $\tilde{M}$ is said to be almost-complex or holomorphic, if its differential intertwines the two almostcomplex structures. On any almost-Hermitian manifold there is a preferred choice of connection, the so-called canonical connection, that generalizes the Chern connection in the integrable case. In general, it is different from the Levi-Civita connection, so it has non-trivial torsion, but is more suited for analytic questions [24]. From now on, all geometric quantities (Ricci and bisectional curvature, torsion, etc.) will be the ones of the canonical connection. With this setup, we have the following Schwarz lemma (see Section 2 for notation).

Theorem 1.1. Let $\left(M^{2 n}, J, g\right)$ be a complete almost-Hermitian manifold with second Ricci curvature bounded from below by $-K_{1}$, and with torsion and $(2,0)$ part of the curvature bounded. Let $\left(\tilde{M}^{2 \tilde{n}}, \tilde{J}, \tilde{g}\right)$ be an almostHermitian manifold with bisectional curvature bounded from above by $-K_{2}$, $K_{2}>0$. If $f: M \rightarrow \tilde{M}$ is a non-constant almost-complex map, then we must have $K_{1} \geq 0$ and

$$
f^{*} \tilde{g} \leq \frac{K_{1}}{K_{2}} g .
$$

In particular if $K_{1} \leq 0$ then any almost-complex map is constant.

Corollary 1.1. Let $\left(M^{2 n}, J, g\right)$ be a complete almost-Hermitian manifold with non-negative second Ricci curvature and with torsion and $(2,0)$ part of the curvature bounded. Then $M$ does not admit any non-constant bounded $J$-holomorphic function $f: M \rightarrow \mathbb{C}$.

Notice that when $M$ is compact the assumptions of bounded torsion and $(2,0)$ part of the curvature are automatically satisfied. Also, while almostcomplex maps between Kähler manifolds are always harmonic [16], this is no longer true for general almost-complex manifolds (see $[8,(9.11)]$ ), so that the results of [10] do not apply in our situation.

Next we assume that $M$ and $\tilde{M}$ have the same dimension. A map $f: M \rightarrow \tilde{M}$ is called non-degenerate if $f^{*} d V_{\tilde{g}}$ is a volume form on $M$, and totally degenerate if $f^{*} d V_{\tilde{g}}$ vanishes identically. Then we have the following Schwarz lemma for the volume forms.

Theorem 1.2. Let $\left(M^{2 n}, J, g\right)$ be a complete almost-Hermitian manifold with second Ricci curvature bounded from below, with torsion and $(2,0)$ part 
of the curvature bounded, and with scalar curvature bounded from below by $-n K_{1}$. Let $\left(\tilde{M}^{2 n}, \tilde{J}, \tilde{g}\right)$ be an almost-Hermitian manifold of the same dimension $2 n$ with first Ricci curvature bounded from above by $-K_{2}, K_{2}>0$. If $f: M \rightarrow \tilde{M}$ is a non-degenerate almost-complex map, then we must have $K_{1} \geq 0$ and

$$
f^{*} d V_{\tilde{g}} \leq\left(\frac{K_{1}}{K_{2}}\right)^{n} d V_{g} .
$$

In particular if $K_{1} \leq 0$ then any almost-complex map is totally degenerate.

As an application of the Schwarz lemma, we study the geometry of the product of two (non-trivial) almost-complex manifolds. A classical theorem of Preissman implies that a compact Riemannian manifold with negative sectional curvature cannot be topologically a product manifold. For Kähler manifolds the notion of bisectional curvature is more natural, and it is easy to see that a compact Kähler manifold with negative bisectional curvature cannot be the product of two non-trivial complex manifolds (this is because the negativity of the curvature implies that the cotangent bundle is ample). When the two factors are allowed to be non-compact, there are similar results due to Yang, Zheng and Seshadri ([22,26, 29]). In [23] it is proved that the product of two complex manifolds does not admit any complete Hermitian metric with bounded torsion and bisectional curvature bounded between two negative constants. It is natural to expect that such a result should hold in the almost-complex case, and this is precisely what we prove.

Theorem 1.3. Let $M=X \times Y$ be the product of two almost-complex manifolds of positive dimensions. Then $M$ does not admit any complete almostHermitian metric with torsion and $(2,0)$ part of the curvature bounded and with bisectional curvature bounded between two negative constants.

Corollary 1.2. The product of two compact non-trivial almost-complex manifolds does not admit any almost-Hermitian metric with negative bisectional curvature.

Let us stress that here the bisectional curvature is the one of the canonical connection, and in general is different from the one of the Levi-Civita connection (as defined in [12], for example). Nevertheless, this curvature is more natural on almost-Hermitian manifolds (see the discussion after Lemma 3.2).

The proof of the Schwarz lemma employs Cartan's formalism of moving frames and the canonical connection, as in [24]. To deal with the case 
of non-compact manifolds, we generalize Yau's maximum principle [27] to our situation. The proof of this requires a suitable Laplacian comparison theorem for almost-Hermitian manifolds. This is the key technical tool and is proved along the lines of the classical Laplacian comparison, but using local holomorphic discs instead of complex coordinates that are not available, and keeping carefully track of the torsion. The proof of Theorem 1.3 follows the argument in [23], once the Schwarz lemma and the maximum principle hold. The paper is organized as follows: in Section 2, we give some background on almost-Hermitian metrics and the canonical connection. In Section 3, we study the Laplacian of the canonical connection. In Section 4, we prove the Laplacian comparison theorem and the maximum principle. In Section 5, we give a proof of Theorems 1.1 and 1.2. Finally, Theorem 1.3 is proved in Section 6 .

\section{Almost-Hermitian manifolds and the canonical connection}

In this section, we give some background on almost-Hermitian manifolds, the canonical connection and its torsion and curvature. Some of the exposition follows [24, Section 2].

Let $(M, J, g)$ be an almost-Hermitian manifold of dimension $2 n$. Namely, $J$ is an almost complex structure on $M$ and $g$ is a Riemannian metric satisfying

$$
g(J X, J Y)=g(X, Y),
$$

for all tangent vectors $X$ and $Y$. Write $T_{p}^{\mathbb{R}} M$ for the (real) tangent space of $M$ at a point $p$. In the following we will drop the subscript $p$. Denote the complexified tangent space by $T^{\mathbb{C}} M=T^{\mathbb{R}} M \otimes \mathbb{C}$. Extending $g$ and $J$ linearly to $T^{\mathbb{C}} M$, we see that the complexified tangent space can be decomposed as

$$
T^{\mathbb{C}} M=T^{\prime} M \oplus T^{\prime \prime} M,
$$

where $T^{\prime} M$ and $T^{\prime \prime} M$ are the eigenspaces of $J$ corresponding to eigenvalues $\sqrt{-1}$ and $-\sqrt{-1}$, respectively. $T^{\prime} M$ and $T^{\prime \prime} M$ are complex vector spaces of dimension $n$, which inherit a Hermitian metric induced by $g$. Note that by extending $J$ to forms, we can uniquely decompose $m$-forms into $(p, q)$-forms for each $p, q$ with $p+q=m$. The real tangent bundle $T^{\mathbb{R}} M$ can be identified with $T^{\prime} M$ in a natural way, by sending a vector $X_{\mathbb{R}}$ to $X=\frac{1}{\sqrt{2}}\left(X_{\mathbb{R}}-\sqrt{-1} J X_{\mathbb{R}}\right)$. This identification is an isomorphism of complex vector bundles, and an isometry. From now on, we will write $g$ for the induced Hermitian metric on $T^{\prime} M$, and $d V_{g}$ for its corresponding volume element. Choose a local unitary frame $\left\{e_{1}, \ldots, e_{n}\right\}$ for $T^{\prime} M$, and 
let $\left\{\theta^{1}, \ldots, \theta^{n}\right\}$ be a dual coframe. Then we can write $g=\theta^{i} \otimes \overline{\theta^{i}}$ and $d V_{g}=(\sqrt{-1})^{n} \theta^{1} \wedge \overline{\theta^{1}} \wedge \cdots \wedge \theta^{n} \wedge \overline{\theta^{n}}$, where here, and henceforth, we are summing over repeated indices.

Let $\nabla$ be an affine connection on $T^{\mathbb{R}} M$, which we extend linearly to $T^{\mathbb{C}} M$. We say that $\nabla$ is an almost-Hermitian connection if

$$
\nabla J=\nabla g=0
$$

It is easy to see that such connections always exist on any almostHermitian manifold, and from now on we shall assume that $\nabla$ satisfies this condition. Observe that $J\left(\nabla e_{i}\right)=\sqrt{-1} \nabla e_{i}$, and hence $\nabla e_{i} \in T^{\prime} M \otimes$ $\left(T^{\mathbb{C}}(M)\right)^{*}$. Then locally there exists a matrix of complex valued 1-forms $\left\{\theta_{i}^{j}\right\}$, called the connection 1 -forms, such that

$$
\nabla e_{i}=\theta_{i}^{j} e_{j}
$$

Applying $\nabla$ to $g\left(e_{i}, \overline{e_{j}}\right)$ and using the condition $\nabla g=0$, we see that $\left\{\theta_{i}^{j}\right\}$ satisfies the skew-Hermitian property

$$
\theta_{i}^{j}+\overline{\theta_{j}^{i}}=0
$$

Now define the torsion $\Theta=\left(\Theta^{1}, \ldots, \Theta^{n}\right)$ of $\nabla$ by

$$
d \theta^{i}=-\theta_{j}^{i} \wedge \theta^{j}+\Theta^{i}, \quad \text { for } i=1, \ldots, n .
$$

Notice that the $\Theta^{i}$ are 2-forms. Equation (2.1) is known as the first structure equation. Define the curvature $\Omega=\left\{\Omega_{j}^{i}\right\}$ of $\nabla$ by

$$
d \theta_{j}^{i}=-\theta_{k}^{i} \wedge \theta_{j}^{k}+\Omega_{j}^{i}
$$

Note that $\left\{\Omega_{j}^{i}\right\}$ is a skew-Hermitian matrix of 2-forms. Equation (2.2) is known as the second structure equation. We have the following lemma (see, e.g., [9]).

Lemma 2.1. There exists a unique almost-Hermitian connection $\nabla$ on $(M, J, g)$ whose torsion $\Theta$ has everywhere vanishing $(1,1)$ part.

We call such a connection the canonical connection. In Riemannian geometry the torsion of a connection $\nabla$ is usually defined by

$$
\nabla_{X} Y=\nabla_{Y} X+[X, Y]+\tau(X, Y)
$$


We will show in Lemma 3.2 that in $T^{\mathbb{C}} M$ the following identity holds:

$$
\tau=2\left(\Theta^{i} e_{i}+\overline{\Theta^{j} e_{j}}\right) .
$$

Define functions $M_{j k}^{i}$ and $N_{\bar{j} \bar{k}}^{i}$ by

$$
\left(\Theta^{i}\right)^{(2,0)}=M_{j k}^{i} \theta^{j} \wedge \theta^{k}, \quad\left(\Theta^{i}\right)^{(0,2)}=N_{\bar{j}}^{i} \overline{\theta^{j}} \wedge \overline{\theta^{k}}
$$

with $M_{j k}^{i}=-M_{k j}^{i}$ and $N_{\bar{j} \bar{k}}^{i}=-N_{\bar{k} \bar{j}}^{i}$. Define $R_{i k \bar{\ell}}^{j}, K_{j k \ell}^{i}$ and $K_{j \bar{k} \bar{\ell}}^{i}$ by

$$
\begin{aligned}
& \left(\Omega_{i}^{j}\right)^{(1,1)}=R_{i k \bar{\ell}}^{j} \theta^{k} \wedge \overline{\theta^{\ell}} \\
& \left(\Omega_{j}^{i}\right)^{(2,0)}=K_{j k \ell}^{i} \theta^{k} \wedge \theta^{\ell} \\
& \left(\Omega_{j}^{i}\right)^{(0,2)}=K_{j \bar{k} \bar{\ell}}^{i} \overline{\theta^{k}} \wedge \overline{\theta^{\ell}}
\end{aligned}
$$

with $K_{j k \ell}^{i}=-K_{j \ell k}^{i}$ and $K_{j \bar{k} \bar{\ell}}^{i}=-K_{j \bar{\ell} \bar{k}}^{i}$. The fact that $\left\{\Omega_{j}^{i}\right\}$ is skew-Hermitian implies that

$$
K_{j k \ell}^{i}=\overline{K_{i \bar{\ell} \bar{k}}^{j}}, \quad R_{j k \bar{\ell}}^{i}=\overline{R_{i \ell \bar{k}}^{j}} .
$$

If $X, Y$ are two $(1,0)$ vectors, we define

$$
B(X, Y)=\frac{R_{i k \bar{\ell}}^{j} X^{i} \overline{X^{j}} Y^{k} \overline{Y^{\ell}}}{\|X\|^{2}\|Y\|^{2}}
$$

to be the bisectional curvature of the canonical connection in the directions $X$ and $Y$, which is a real number. We define the first Ricci curvature, the second Ricci curvature and the scalar curvature of the canonical connection to be the tensors $R_{k \bar{\ell}}=R_{i k \bar{\ell}}^{i}, R_{k \bar{\ell}}^{\prime}=R_{k i \bar{i}}^{\ell}$ and $R=R_{k \bar{k}}=R_{k \bar{k}}^{\prime}$, respectively. Applying the exterior derivative to the first structure equation, we obtain the first Bianchi identity,

$$
d \Theta^{i}=\Omega_{j}^{i} \wedge \theta^{j}-\theta_{j}^{i} \wedge \Theta^{j}
$$

If we define $M_{j k, p}^{i}, M_{j k, \bar{p}}^{i}$ by

$$
d M_{j k}^{i}+\theta_{p}^{i} M_{j k}^{p}-M_{p k}^{i} \theta_{j}^{p}-M_{j p}^{i} \theta_{k}^{p}=M_{j k, p}^{i} \theta^{p}+M_{j k, \bar{p}}^{i} \overline{\theta^{p}}
$$

and $N_{\bar{j}}^{i} \bar{k}, p_{\text {and }} N_{\bar{j} \bar{k}, \bar{p}}^{i}$ by

$$
d N_{\bar{j} \bar{k}}^{i}+\theta_{p}^{i} N_{\bar{j} \bar{k}}^{p}-N_{\bar{p}}^{i} \bar{k} \overline{\theta_{j}^{p}}-N_{\bar{j} \bar{p}}^{i} \overline{\theta_{k}^{p}}=N_{\bar{j} \bar{k}, p}^{i} \theta^{p}+N_{\bar{j} \bar{k}, \bar{p}}^{i} \overline{\theta^{p}},
$$


then the first Bianchi identity implies that (see, e.g., [24, Section 2.3])

$$
2 M_{p j}^{i} N_{\bar{k} \bar{\ell}}^{p}+N_{\bar{k} \bar{\ell}, j}^{i}=K_{j \bar{k} \bar{\ell}}^{i}
$$

We say that the bisectional curvature is bounded above by $A$ if

$$
B(X, Y) \leq A
$$

holds for all $X, Y \in T^{\prime} M$. The first Ricci curvature is bounded below by a constant $-A_{1}$ if

$$
R_{k \bar{\ell}} X^{k} \overline{X^{\ell}} \geq-A_{1}\|X\|^{2}
$$

holds for all $X \in T^{\prime} M$, and the same for the second Ricci curvature. The torsion is bounded by $A_{2}>0$ if

$$
\|\tau(X, Y)\| \leq A_{2}\|X\|\|Y\|
$$

holds for all $X, Y \in T^{\prime} M$, and the $(2,0)$ part of the curvature is bounded by $A_{3}$ if

$$
\left|K_{j k \ell}^{i} \overline{X^{i}} Y^{j} Y^{k} X^{\ell}\right| \leq A_{3}\|X\|^{2}\|Y\|^{2}
$$

\section{The canonical Laplacian}

In this section, we study the Laplacian of the canonical connection, and relate it to the standard Laplacian of the Levi-Civita connection. Again, part of the exposition follows [24].

Let $\nabla$ be the canonical connection of $(M, J, g)$, and $u$ be a function on $M$. We define the canonical Laplacian $\Delta$ of $u$ by

$$
\Delta u=\sum_{i}\left((\nabla \nabla u)\left(e_{i}, \overline{e_{i}}\right)+(\nabla \nabla u)\left(\overline{e_{i}}, e_{i}\right)\right) .
$$

This expression is independent of the choice of unitary frame. Another way to define $\Delta u$ is as follows. Let $\left\{\nu_{1}, \ldots, \nu_{2 n}\right\}$ be a real local orthonormal frame for $g$ and set

$$
\Delta u=\sum_{A=1}^{2 n}(\nabla \nabla u)\left(\nu_{A}, \nu_{A}\right)
$$

Clearly this expression is independent of the choice of frame and coincides with the one above. Now define $u_{i}$ and $u_{\bar{i}}$ by

$$
d u=u_{i} \theta^{i}+u_{\bar{i}} \overline{\theta^{i}} .
$$


Writing $\partial u$ and $\bar{\partial} u$ for the $(1,0)$ and $(0,1)$ parts of $d u$, respectively, we see that $\partial u=u_{i} \theta^{i}$ and $\bar{\partial} u=u_{\bar{i}} \bar{\theta}^{i}$. Define $u_{i k}, u_{i \bar{k}}, u_{\bar{i} k}$ and $u_{\bar{i} \bar{k}}$ by

$$
\begin{aligned}
& d u_{i}-u_{j} \theta_{i}^{j}=u_{i k} \theta^{k}+u_{i \bar{k}} \overline{\theta^{k}} \\
& d u_{\bar{i}}-u_{\bar{j}} \theta_{i}^{j}=u_{\bar{i} k} \theta^{k}+u_{\bar{i} \bar{k}} \overline{\theta^{k}} .
\end{aligned}
$$

The following lemma is proved in [24].

\section{Lemma 3.1.}

$$
\begin{aligned}
\Delta u & =2 \sum_{i} u_{i \bar{i}} \\
& =-2 \sum_{i}(d \partial u)^{(1,1)}\left(e_{i}, \overline{e_{i}}\right) \\
& =2 \sum_{i}(d \bar{\partial} u)^{(1,1)}\left(e_{i}, \overline{e_{i}}\right) \\
& =\sqrt{-1} \sum_{i}(d(J d u))^{(1,1)}\left(e_{i}, \overline{e_{i}}\right),
\end{aligned}
$$

where $J$ acts on a 1-form $\alpha$ by $(J \alpha)(X)=\alpha(J(X))$ for a vector $X$.

We now want to relate the canonical Laplacian to the standard LeviCivita Laplacian. In general they are different, and their precise relation is given by the following lemma.

Lemma 3.2. The Laplacian of the Levi-Civita connection of $g$ acting on a function $u$ is equal to

$$
\Delta u+2 M_{j i}^{i} u_{\bar{j}}+2 \overline{M_{j i}^{i}} u_{j}
$$

Proof. The Laplacian of the Levi-Civita connection applied to $u$ is given by the trace of the map $F: T^{\mathbb{R}} M \rightarrow T^{\mathbb{R}} M$ defined by

$$
F(X)=\nabla_{X}\left(\operatorname{grad}_{g} u\right)+\tau\left(\operatorname{grad}_{g} u, X\right),
$$

(see, for example, [15, p. 282]) where $\nabla$ is the canonical connection and $\tau$ is its torsion, as defined in (2.3). To prove the lemma, it is enough to show that (2.4) holds. We verify this for $X, Y \in T^{\prime} M$ first. Define functions 
$X_{p}^{i}, X_{\bar{p}}^{i}, X_{p q}^{i}, X_{p \bar{q}}^{i}, X_{\bar{p} q}^{i}, X_{\bar{p} \bar{q}}^{i}$ by

$$
\begin{gathered}
d X^{i}+X^{j} \theta_{j}^{i}=X_{p}^{i} \theta^{p}+X_{\bar{p}}^{i} \overline{\theta^{p}} \\
d X_{p}^{i}+X_{p}^{j} \theta_{j}^{i}-X_{j}^{i} \theta_{p}^{j}=X_{p q}^{i} \theta^{q}+X_{p \bar{q}}^{i} \overline{\theta^{q}} \\
d X_{\bar{p}}^{i}+X_{\bar{p}}^{j} \theta_{j}^{i}-X_{\bar{j}}^{i} \theta_{p}^{j}=X_{\bar{p} q}^{i} \theta^{q}+X_{\bar{p}}^{i} \overline{\theta^{q}}
\end{gathered}
$$

and similarly for $Y$. Then we have

$$
\begin{aligned}
\nabla_{X} Y & =X^{p} \nabla_{e_{p}}\left(Y^{i} e_{i}\right)=X^{p}\left\langle e_{p}, d Y^{i}\right\rangle e_{i}+X^{p} Y^{i}\left\langle\theta_{i}^{k}, e_{p}\right\rangle e_{k} \\
& =-X^{p} Y^{k}\left\langle\theta_{k}^{i}, e_{p}\right\rangle e_{i}+X^{p} Y_{p}^{i} e_{i}+X^{p} Y^{i}\left\langle\theta_{i}^{k}, e_{p}\right\rangle e_{k}=X^{p} Y_{p}^{i} e_{i}
\end{aligned}
$$

Here and in the following $\langle\cdot, \cdot\rangle$ denotes the pairing between vectors and 1-forms. Moreover

$$
\begin{aligned}
& -\left\langle\theta_{\ell}^{i}, X\right\rangle\left\langle\theta^{\ell}, Y\right\rangle+\left\langle\theta_{\ell}^{i}, Y\right\rangle\left\langle\theta^{\ell}, X\right\rangle+2 \Theta^{i}(X, Y)=2 d \theta^{i}(X, Y) \\
& =X\left\langle\theta^{i}, Y\right\rangle-Y\left\langle\theta^{i}, X\right\rangle-\left\langle\theta^{i},[X, Y]\right\rangle \\
& =X^{j}\left\langle e_{j}, d Y^{i}\right\rangle-Y^{k}\left\langle e_{k}, d X^{i}\right\rangle-\left\langle\theta^{i},[X, Y]\right\rangle \\
& =-X^{j} Y^{k}\left\langle\theta_{k}^{i}, e_{j}\right\rangle+Y^{k} X^{j}\left\langle\theta_{j}^{i}, e_{k}\right\rangle+X^{j} Y_{j}^{i}-Y^{k} X_{k}^{i}-\left\langle\theta^{i},[X, Y]\right\rangle \\
& =-\left\langle\theta_{k}^{i}, X\right\rangle\left\langle\theta^{k}, Y\right\rangle+\left\langle\theta_{j}^{i}, Y\right\rangle\left\langle\theta^{j}, X\right\rangle+\left\langle\theta^{i}, \nabla_{X} Y-\nabla_{Y} X-[X, Y]\right\rangle,
\end{aligned}
$$

which shows that the $e_{i}$ component of $\tau$ is $2 \Theta^{i}$. Similarly

$$
2 \overline{\Theta^{i}}(X, Y)=2 d \overline{\theta^{i}}(X, Y)=-\left\langle\overline{\theta^{i}},[X, Y]\right\rangle=\left\langle\overline{\theta^{i}}, \nabla_{X} Y-\nabla_{Y} X-[X, Y]\right\rangle \text {, }
$$

so that the $\overline{e_{i}}$ component of $\tau$ is $2 \overline{\Theta^{i}}$.

Now we take $X \in T^{\prime} M, Y \in T^{\prime \prime} M$ (the case when $X, Y \in T^{\prime \prime} M$ is the same as the one above). Then

$$
\begin{aligned}
\nabla_{X} Y & =X^{p} \nabla_{e_{p}}\left(\overline{Y^{i} e_{i}}\right)=X^{p}\left\langle e_{p}, d \overline{Y^{i}}\right\rangle \overline{e_{i}}+X^{p} \overline{Y^{i}}\left\langle\overline{\theta_{i}^{k}}, e_{p}\right\rangle \overline{e_{k}} \\
& =-X^{p} \overline{Y^{k}}\left\langle\overline{\theta_{k}^{i}}, e_{p}\right\rangle \overline{e_{i}}+X^{p} \overline{Y_{\bar{p}}^{i} e_{i}}+X^{p} \overline{Y^{i}}\left\langle\overline{\theta_{i}^{k}}, e_{p}\right\rangle \overline{e_{k}}=X^{p} \overline{Y_{\bar{p}}^{i} e_{i}}
\end{aligned}
$$

and similarly $\nabla_{Y} X=\overline{Y^{p}} X_{\bar{p}}^{i} e_{i}$. Then

$$
\begin{aligned}
& \left\langle\theta_{\ell}^{i}, Y\right\rangle\left\langle\theta^{\ell}, X\right\rangle+2 \Theta^{i}(X, Y)=2 d \theta^{i}(X, Y)=-Y\left\langle\theta^{i}, X\right\rangle-\left\langle\theta^{i},[X, Y]\right\rangle \\
& \quad=-\overline{Y^{k}}\left\langle\overline{e_{k}}, d X^{i}\right\rangle-\left\langle\theta^{i},[X, Y]\right\rangle=\overline{Y^{k}} X^{j}\left\langle\theta_{j}^{i}, \overline{e_{k}}\right\rangle-\overline{Y^{k}} X_{\bar{k}}^{i}-\left\langle\theta^{i},[X, Y]\right\rangle \\
& \quad=\left\langle\theta_{j}^{i}, Y\right\rangle\left\langle\theta^{j}, X\right\rangle+\left\langle\theta^{i}, \nabla_{X} Y-\nabla_{Y} X-[X, Y]\right\rangle,
\end{aligned}
$$

which shows again that the $e_{i}$ component of $\tau$ is $2 \Theta^{i}$, and the verification of the $\overline{e_{i}}$ component is analogous. 
A corollary of this is the following observation: if $u$ achieves its infimum at a point $x \in M$, then $\Delta u(x) \geq 0$. We will use this remark later.

Along the same lines as in Lemma 3.2, it is easy to verify that the bisectional curvature satisfies

$$
\frac{1}{2} B(X, Y)\|X\|^{2}\|Y\|^{2}=R(V, J V, J W, W),
$$

where $R$ is the (real) Riemann curvature tensor of the canonical connection, and $V=\frac{1}{\sqrt{2}}(X+\bar{X}), W=\frac{1}{\sqrt{2}}(Y+\bar{Y})$ are two real tangent vectors. This quantity is in general different from

$$
R^{\mathrm{LC}}(V, J V, J W, W)
$$

where $R^{\mathrm{LC}}$ is the curvature of the Levi-Civita connection. This is usually referred to as the holomorphic bisectional curvature [12], but is not very natural on a general almost-Hermitian manifolds. In fact, it is not hard to see [14] that the bisectional curvature of the canonical connection of an almost-complex submanifold is always less than the one of the ambient space, but this fails, in general, for the Levi-Civita connection (see [12, Proposition 10.1]). The two quantities obviously agree on a Kähler manifold.

Let $(M, J, g)$ and $(\tilde{M}, \tilde{J}, \tilde{g})$ be two almost-Hermitian manifolds of dimensions $2 n$ and $2 \tilde{n}$, respectively, and let $f: M \rightarrow \tilde{M}$ be an almost-complex mapping, which means that

$$
\tilde{J} \circ f_{*}=f_{*} \circ J
$$

We will also say that $f$ is $(J, \tilde{J})$-holomorphic. Then we have the following invariance property.

Lemma 3.3. For any function $u$ on $\tilde{M}$ we have

$$
f^{*} d(\tilde{J} d u)=d(J d(u \circ f))
$$

Proof. If $X$ is vector tangent to $M$ then

$$
\begin{aligned}
\left\langle f^{*} \tilde{J} d u, X\right\rangle & =\left\langle\tilde{J} d u, f_{*} X\right\rangle=\left\langle d u,\left(\tilde{J} \circ f_{*}\right)(X)\right\rangle=\left\langle d u, f_{*} J X\right\rangle \\
& =\langle d(u \circ f), J X\rangle=\langle J d(u \circ f), X\rangle,
\end{aligned}
$$

and taking the exterior derivative we get the conclusion. 


\section{The maximum principle}

In this section, we prove a version of Yau's generalized maximum principle [27] for almost-Hermitian manifolds. The key tool is a Laplacian comparison theorem, whose analog in Riemannian and Kähler geometry is standard [21]. It was extended to Hermitian manifolds in [4] and we will show that it still holds in our more general setting.

The first result is as follows.

Theorem 4.1. Let $(M, J, g)$ be a complete almost-Hermitian manifold with second Ricci curvature bounded below and with torsion and $(2,0)$ part of the curvature bounded. Let $u$ be a non-negative function that is not identically zero and satisfies

$$
\Delta u \geq A u^{1+\alpha}-B u
$$

where $\alpha, A>0$. Then $u$ is bounded above, $B \geq 0$, and

$$
\sup _{M} u \leq\left(\frac{B}{A}\right)^{1 / \alpha} \text {. }
$$

This can be proved exactly in the same way as in [28], once we have the following proposition.

Proposition 4.1 (Maximum principle). Let $(M, J, g)$ be a complete almost-Hermitian manifold with second Ricci curvature bounded below and with torsion and $(2,0)$ part of the curvature bounded. Let $u$ be a real function that is bounded from below. Then given any $\varepsilon>0$ there exists a point $x_{\varepsilon} \in M$ such that

$$
\begin{aligned}
\liminf _{\varepsilon \rightarrow 0} u\left(x_{\varepsilon}\right) & =\inf _{M} u, \\
|\nabla u|\left(x_{\varepsilon}\right) & \leq \varepsilon \\
\Delta u\left(x_{\varepsilon}\right) & \geq-\varepsilon .
\end{aligned}
$$

The proof of this follows the one in [27] and relies on the following theorem.

Theorem 4.2 (Laplacian comparison). Let $(M, J, g)$ be a complete almost-Hermitian manifold with second Ricci curvature bounded below by $-A_{1}$, torsion bounded by $A_{2}$ and $(2,0)$ part of the curvature bounded 
by $A_{3}$. Let $\rho$ be the distance from a fixed point $o \in M$. Then at any point where $\rho$ is smooth we have

$$
\Delta \rho \leq \frac{2 n}{\rho}+C,
$$

where $C$ depends only on $A_{1}, A_{2}, A_{3}$ and the dimension of $M$. Moreover, this holds on the whole of $M$ in the sense of distributions.

Proof. Fix a point $x \in M$ outside the cut locus of $o$, and a minimal unitspeed geodesic $\gamma:[0, \rho(x)] \rightarrow M$ from $o$ to $x$. Let $D \subset \mathbb{C}$ be the unit disc, $z$ be the coordinate on $D$ and $e=\partial /\left.\partial z\right|_{z=0}$ be the tangent vector at the origin. If $v \in T_{x}^{\prime} M$ is small enough then Proposition 1.1 in [13] (see also [19]) gives a $J$-holomorphic map $F: D \rightarrow M$ with $F(0)=x$ and $F_{*}(e)=v$, which depends smoothly on $x$ and $v$. Now extend $v$ to a section $v(t)$ of $T^{\prime} M$ along $\gamma$, that is small enough and vanishes at $o$. Using [13, Theorem A1] and the compactness of the support of $\gamma$, we can extend $F$ to a smooth family $F_{t}: D \rightarrow M$ of $J$-holomorphic discs, with the properties that $F_{\rho(x)}=F$, $F_{t}(0)=\gamma(t), F_{t *}(e)=v(t)$ and $F_{0}(z)=o$. We will write $F(t, z)=F_{t}(z)$ so that we have a map $F:[0, \rho(x)] \times D \rightarrow M$. Notice that we can also allow $v=\gamma^{\prime}(\rho(x))$. The vector $F_{*}(\partial / \partial t)$ belongs to $T^{\mathbb{R}} M \subset T^{\mathbb{C}} M$, and so it can be written as $T+\bar{T}$ where $T \in T^{\prime} M$. Moreover, the fact that $F_{t}$ is $J$-holomorphic implies that the vector $S=F_{*}(\partial / \partial z)$ belongs to $T^{\prime} M$. Notice that both $T$ and $S$ depend on $(t, z)$ and that $S(t, 0)=v(t),(T+\bar{T})(t, 0)=$ $\gamma^{\prime}(t)$. The map $F$ that we just constructed should be thought of as a $J$-holomorphic variation of $\gamma$, and we are going to compute the second variation of the arclength. This is the function $L: D \rightarrow \mathbb{R}$ defined by

$$
L(z)=\sqrt{2} \int_{0}^{\rho(x)}\|T(t, z)\| d t,
$$

which is just the length of the curve $t \mapsto F(t, z)$, that goes from $o$ to $F(\rho(x), z)$, a point near $x$. Fixing for a moment $(t, z)$, we can take a local unitary frame $\left\{e_{i}\right\}$ near $F(t, z)$ and write $T=T^{i} e_{i}, S=S^{j} e_{j}$. Then

$$
\begin{aligned}
d(\|T\|)=d\left(T^{i} \overline{T^{i}}\right)^{1 / 2} & =\frac{1}{2\|T\|}\left(T_{p}^{i} \overline{T^{i}} \theta^{p}+T_{\bar{p}}^{i} \overline{T^{i} \theta^{p}}+T^{i} \overline{T_{p}^{i} \theta^{p}}+T^{i} \overline{T_{\bar{p}}^{i}} \theta^{p}\right), \\
\frac{\partial}{\partial z}\|T\| & =\langle d\|T\|, S\rangle=\frac{T_{p}^{i} \overline{T^{i}} S^{p}+T^{i} \overline{T_{\bar{p}}^{i}} S^{p}}{2\|T\|}
\end{aligned}
$$


The term $T^{i} \overline{T_{\bar{p}}^{i}} S^{p}$ can be computed as follows:

$$
[T+\bar{T}, \bar{S}]=F_{*}([\partial / \partial t, \partial / \partial \bar{z}])=0,
$$

and so $[T, \bar{S}]=[\bar{S}, \bar{T}]$. Combining (2.3), (2.4), (3.9) and (3.10) we get

$$
\theta^{i}([T, \bar{S}])=-\theta^{i}\left(\nabla_{\bar{S}} T\right)=-\overline{S^{p}} T_{\bar{p}}^{i}
$$

because $\Theta^{i}$ has no $(1,1)$ component. But we also have

$$
\theta^{i}([T, \bar{S}])=\theta^{i}([\bar{S}, \bar{T}])=-2 \Theta^{i}(\bar{S}, \bar{T})=-2 N_{\bar{j}}^{i} \bar{k} \overline{S^{j} T^{k}},
$$

and so

$$
\overline{S^{p}} T_{\bar{p}}^{i}=2 N_{\bar{j}}^{i} \overline{S^{j} T^{k}}
$$

By the same token, $\theta^{i}([T, S])=T^{j} S_{j}^{i}-S^{j} T_{j}^{i}-2 M_{j k}^{i} T^{j} S^{k}$, but we also have that $\theta^{i}([T, S])=\theta^{i}([S, \bar{T}])=-\overline{T^{j}} S_{j}^{i}$, and so

$$
S^{j} T_{j}^{i}=T^{j} S_{j}^{i}+\overline{T^{j}} S_{j}^{i}-2 M_{j k}^{i} T^{j} S^{k}=\left\langle\theta^{i}, \nabla_{T+\bar{T}} S-\tau(T, S)\right\rangle .
$$

Also, $[S, \bar{S}]=F_{*}([\partial / \partial z, \partial / \partial \bar{z}])=0$ implies $\overline{S^{p}} S_{\bar{p}}^{i}=0$. Using this, we differentiate (4.3) once more and we get

$$
\begin{aligned}
\frac{\partial^{2}}{\partial z \partial \bar{z}}\|T\|= & \left\langle d\left(\frac{T_{p}^{i} \overline{T^{i}} S^{p}+2 \overline{N_{\bar{j}}^{i}} T^{i} S^{j} T^{k}}{2\|T\|}\right), \bar{S}\right\rangle \\
= & -\frac{\left|T_{p}^{i} \overline{T^{i}} S^{p}+2 \overline{N_{\bar{j}}^{i}} T^{i} S^{j} T^{k}\right|^{2}}{4\|T\|^{3}}+\frac{T_{p \bar{q}}^{i} \overline{T^{i}} S^{p} \overline{S^{q}}+T_{p}^{i} \overline{T_{q}^{i}} S^{p} \overline{S^{q}}}{2\|T\|} \\
& +\frac{2 \overline{N_{\bar{j}}^{i}, q} T^{i} S^{j} T^{k} \overline{S^{q}}+2 \overline{N_{\bar{j}}^{i}} T_{\bar{q}}^{i} S^{j} T^{k} \overline{S^{q}}+2 \overline{N_{\bar{j}}^{i} \bar{k}} T^{i} S^{j} T_{\bar{q}}^{k} \overline{S^{q}}}{2\|T\|} .
\end{aligned}
$$

To deal with the term $T_{p \bar{q}}^{i} \overline{T^{i}} S^{p} \overline{S^{q}}$ we take the exterior derivative of (3.6) and using (3.7), (3.8) we get

$$
T^{j} \Omega_{j}^{i}=T_{p q}^{i} \theta^{q} \wedge \theta^{p}+T_{p \bar{q}}^{i} \overline{\theta^{q}} \wedge \theta^{p}+T_{p}^{i} \Theta^{p}+T_{\bar{p} q}^{i} \theta^{q} \wedge \overline{\theta^{p}}+T_{\bar{p}}^{i} \overline{\theta^{q}} \wedge \overline{\theta^{p}}+T_{\bar{p}}^{i} \overline{\Theta^{p}}
$$

whose $(1,1)$ part gives $T_{p \bar{q}}^{i}=T_{\bar{q} p}^{i}-T^{j} R_{j p \bar{q}}^{i}$, and so

$$
T_{p \bar{q}}^{i} \overline{T^{i}} S^{p} \overline{S^{q}}=T_{\bar{q} p}^{i} \overline{T^{i}} S^{p} \overline{S^{q}}-T^{j} \overline{T^{i}} S^{p} \overline{S^{q}} R_{j p \bar{q}}^{i}
$$


The term $T_{\bar{q} p}^{i} \overline{T^{i}} S^{p} \overline{S^{q}}$ can now be computed as follows:

$$
\begin{aligned}
0= & \left\langle d\left(\overline{S^{q}} T_{\bar{q}}^{i} \overline{T^{i}}-2 N_{\bar{j}}^{i} \overline{S^{j} T^{k} T^{i}}\right), S\right\rangle=\overline{S^{q}} T_{\bar{q} p}^{i} \overline{T^{i}} S^{p}+\overline{S^{q}} T_{\bar{q}}^{i} \overline{T_{\bar{p}}^{i}} S^{p} \\
& -2 N_{\bar{j} \bar{k}, p}^{i} \overline{S^{j} T^{k} T^{i}} S^{p}-2 N_{\bar{j} \bar{k}}^{i} \overline{S^{j} T_{\bar{p}}^{k} T^{i}} S^{p}-2 N_{\bar{j} \bar{k}}^{i} \overline{S^{j} T^{k} T_{\bar{p}}^{i}} S^{p},
\end{aligned}
$$

and using (4.4) we get

$$
T_{\bar{q} p}^{i} \overline{T^{i}} S^{p} \overline{S^{q}}=2 N_{\bar{j} \bar{k}, p^{i}}^{i} \overline{S^{j} T^{k} T^{i}} S^{p}+2 N_{\bar{j} \bar{k}}^{i} \overline{S^{j} T_{\bar{p}}^{k} T^{i}} S^{p} .
$$

Combining (4.6), (4.7), (4.8), (4.5), (4.4), (2.10), (2.5) and (2.6) we get

$$
\begin{aligned}
\frac{\partial^{2}}{\partial z \partial \bar{z}}\|T\| & \\
\leq & \frac{-B(T, S)\|T\|^{2}\|S\|^{2}+\left|\left\langle\theta^{i}, \nabla_{T+\bar{T}} S-\tau(T, S)\right\rangle\right|^{2}+\left|\left\langle\theta^{i}, \tau(\bar{S}, \bar{T})\right\rangle\right|^{2}}{2\|T\|} \\
& +\frac{4 \operatorname{Re}\left(\overline{K_{i k j}^{p} S^{j} T^{k} T^{i}} S^{p}-2 M_{q p}^{i} N_{\bar{j} \bar{k}}^{q} \overline{S^{j} T^{k} T^{i}} S^{p}+N_{\bar{j} \bar{k}}^{i} \overline{S^{j} T^{i} N_{\bar{p}}^{k}} S^{p} T^{q}\right)}{2\|T\|} \\
& \leq \frac{-B(T, S)\|T\|^{2}\|S\|^{2}+\left\|\nabla_{T+\bar{T}} S-\tau(T+\bar{T}, S)\right\|^{2}}{2\|T\|} \\
& +\frac{\left(13 A_{2}^{2}+4 A_{3}\right)\|S\|^{2}\|T\|^{2}}{2\|T\|} .
\end{aligned}
$$

All the terms on the right-hand side are tensorial, and hence independent of the choice of unitary frame. Combining this with (4.2) and setting $z=0$ we finally get

$$
\begin{aligned}
\left.\frac{\partial^{2}}{\partial z \partial \bar{z}} L(z)\right|_{z=0} \leq & \frac{1}{2} \int_{0}^{\rho(x)}\left\|\nabla_{\gamma^{\prime}(t)} v(t)+\tau\left(v(t), \gamma^{\prime}(t)\right)\right\|^{2} d t \\
& +\frac{1}{2} \int_{0}^{\rho(x)}\left(C-B\left(\gamma^{\prime}(t), v(t)\right)\right)\|v(t)\|^{2} d t
\end{aligned}
$$

where $C=13 A_{2}^{2}+4 A_{3}$. Notice that the right-hand side is homogeneous of degree 2 in $v(t)$, so it does not matter that we had picked $v(t)$ small in the first place. Define $G: D \rightarrow M$ to be the $J$-holomorphic disc $G(z)=$ $F(\rho(x), z)$, originally called $F$, and notice that since $\gamma$ is minimizing we have $L(z) \geq(\rho \circ G)(z)$ and $L(0)=(\rho \circ G)(0)$, hence

$$
\left.\frac{\partial^{2}}{\partial z \partial \bar{z}} L(z)\right|_{z=0} \geq\left.\frac{\partial^{2}}{\partial z \partial \bar{z}}(\rho \circ G)(z)\right|_{z=0} .
$$


But now Lemmas 3.1 and 3.3 imply that

$$
\begin{aligned}
\sqrt{-1} d(J d \rho)^{(1,1)}(v, \bar{v}) & =\sqrt{-1} d\left(J_{\mathbb{C}} d(\rho \circ G)\right)(e, \bar{e}) \\
& =\left.4 \frac{\partial^{2}}{\partial z \partial \bar{z}}(\rho \circ G)(z)\right|_{z=0},
\end{aligned}
$$

where $J_{\mathbb{C}}$ is the standard complex structure on $\mathbb{C}$.

Now we choose $v$ to have unit length, and we choose $v(t)$ to be of the form $v(t)=f(t) w(t)$, where $w(t)$ is the parallel transport with respect to $\nabla$ of $v$ along $\gamma$, and $f(t) \geq 0$ is a smooth increasing function that satisfies $f(0)=0$ and $f(\rho(x))=1$. Then, using (4.9) and the fact that $\nabla g=0$, we get

$$
\begin{aligned}
\left.\frac{\partial^{2}}{\partial z \partial \bar{z}} L(z)\right|_{z=0} \leq & \frac{1}{2} \int_{0}^{\rho(x)}\left\|f^{\prime}(t) w(t)+f(t) \tau\left(w(t), \gamma^{\prime}(t)\right)\right\|^{2} d t \\
& +\frac{1}{2} \int_{0}^{\rho(x)} f(t)^{2}\left(C-B\left(\gamma^{\prime}(t), w(t)\right)\right) d t \\
\leq & \frac{1}{2} \int_{0}^{\rho(x)}\left(f^{\prime}(t)^{2}+2 A_{2} f(t) f^{\prime}(t)+A_{2}^{2} f(t)^{2}\right) d t \\
& +\frac{1}{2} \int_{0}^{\rho(x)} f(t)^{2}\left(C-B\left(\gamma^{\prime}(t), w(t)\right)\right) d t .
\end{aligned}
$$

Now we combine $(4.10),(4.11),(4.12)$ and sum them up when $v$ ranges in $v_{1}, \ldots, v_{n}$, a unitary basis of $T_{x}^{\prime} M$, and using Lemma 3.1 we get

$$
\Delta \rho(x) \leq 2 \int_{0}^{\rho(x)}\left(n f^{\prime}(t)^{2}+2 n A_{2} f(t) f^{\prime}(t)+C^{\prime} f(t)^{2}\right) d t
$$

where $C^{\prime}=n C+A_{1}+n A_{2}^{2}$. Next, following [4], we choose

$$
f(t)=\left(\frac{t}{\rho(x)}\right)^{\alpha}
$$

where $\alpha>1$ will be chosen presently. With this choice we can easily compute that

$$
\Delta \rho(x) \leq \frac{2 n}{\rho(x)}+2 n A_{2}+\frac{2 n(\alpha-1)^{2}}{(2 \alpha-1) \rho(x)}+\frac{2 C^{\prime}}{2 \alpha+1} \rho(x) .
$$

Now we choose $\alpha$, depending on $\rho(x)$, such that the last two terms on the right-hand side are equal. Thus

$$
\frac{n(\alpha-1)^{2}}{(2 \alpha-1) \rho(x)}+\frac{C^{\prime}}{2 \alpha+1} \rho(x)=2 \sqrt{\frac{(\alpha-1)^{2}}{4 \alpha^{2}-1} n C^{\prime}} \leq \sqrt{n C^{\prime}},
$$


which is what we want. Finally, to show that the inequality in the sense of distributions holds on the whole manifold we can just follow the argument in $[21$, p. 7$]$.

Proof of Proposition 4.1. Once we have established the Laplacian comparison theorem, Theorem 4.2, the proof is standard, but we include it for completeness. We will use a trick due to Calabi [2] to avoid the cut locus of $o$. If the infimum of $u$ is attained in the geodesic ball of radius 1 centered at $o$ then there is nothing to prove, so that we may assume that $\rho>1$. Then Theorem 4.2 gives $\Delta \rho \leq C$ for a uniform constant $C$. For any $\varepsilon>0$ the function $u+\varepsilon \rho$ attains its infimum at a point $x_{\varepsilon} \in M$. Let $\gamma$ be a minimal unit-speed geodesic from $o$ to $x_{\varepsilon}, \tilde{x}$ be another point on $\gamma$ and denote by $\tilde{\rho}$ the distance from $\tilde{x}$. Then for any $x \in M$ we have

$$
u(x)+\varepsilon \tilde{\rho}(x)=u(x)+\varepsilon \rho(x)-\varepsilon \rho(x)+\varepsilon \tilde{\rho}(x) \geq u(x)+\varepsilon \rho(x)-\varepsilon \rho(\tilde{x}),
$$

and taking the infimum over $x$ we get

$$
\inf _{M}(u+\varepsilon \tilde{\rho}) \geq u\left(x_{\varepsilon}\right)+\varepsilon \rho\left(x_{\varepsilon}\right)-\varepsilon \rho(\tilde{x})=u\left(x_{\varepsilon}\right)+\varepsilon \tilde{\rho}\left(x_{\varepsilon}\right) .
$$

Hence the function $u+\varepsilon \tilde{\rho}$ also attains its infimum at $x_{\varepsilon}$. But we can now choose $\tilde{x}$ outside the cut locus of $x_{\varepsilon}$, so that $\tilde{\rho}$ is smooth at $x_{\varepsilon}$, and using the remark after Lemma 3.2 we get

$$
\begin{array}{r}
|\nabla u|\left(x_{\varepsilon}\right)=\varepsilon|\nabla \tilde{\rho}|\left(x_{\varepsilon}\right)=\varepsilon, \\
\Delta u\left(x_{\varepsilon}\right) \geq-\varepsilon \Delta \tilde{\rho} \geq-\varepsilon C .
\end{array}
$$

Finally, we check that $\liminf _{\varepsilon \rightarrow 0} u\left(x_{\varepsilon}\right)=\inf _{M} u$. If not, there exist $\bar{x} \in M$ and $\delta>0$ such that $u(\bar{x})<u\left(x_{\varepsilon}\right)-\delta$ for all $\varepsilon$ small. We still have

$$
u(\bar{x})+\varepsilon \rho(\bar{x}) \geq u\left(x_{\varepsilon}\right)+\varepsilon \rho\left(x_{\varepsilon}\right) .
$$

If $\rho\left(x_{\varepsilon}\right)$ is bounded then we can take a convergent subsequence of points and letting $\varepsilon \rightarrow 0$ we get a contradiction. If $\rho\left(x_{\varepsilon}\right)$ is unbounded, we take $\varepsilon$ small so that $\rho\left(x_{\varepsilon}\right)>\rho(\bar{x})$ and get

$$
u\left(x_{\varepsilon}\right)+\varepsilon \rho\left(x_{\varepsilon}\right) \leq u(\bar{x})+\varepsilon \rho(\bar{x})<u\left(x_{\varepsilon}\right)-\delta+\varepsilon \rho\left(x_{\varepsilon}\right),
$$

which again is absurd. 
Proof of Theorem 4.1. Now that we have Proposition 4.1, the argument is exactly the same as in [28] so we will coill just sketch it. One defines a function

$$
v=(u+c)^{-\alpha / 2}
$$

where $c>0$ is fixed. Since $v$ is bounded below we can apply Proposition 4.1 and for any $\varepsilon>0$ we get a point $x_{\varepsilon} \in M$ where we have

$$
A u^{1+\alpha}-B u \leq \Delta u \leq \frac{2}{\alpha}\left((u+c)^{(\alpha+2) / 2}+\varepsilon \frac{\alpha+2}{\alpha}(u+c)^{1+\alpha}\right) \varepsilon
$$

If $\sup _{M} u=+\infty$ then we can let $\varepsilon \rightarrow 0$ in the last inequality and get a contradiction. So $\sup _{M} u<+\infty$ and again letting $\varepsilon \rightarrow 0$ we get the conclusion.

Remark 4.1. Instead of our Theorem 4.2 we could have used the standard Laplacian comparison, as in [27]. This gives a similar result for the Laplacian of the Levi-Civita connection, under the assumption that the Ricci curvature of the Levi-Civita connection is bounded below. Notice that to apply this to our situation we still need the assumption that the torsion be bounded, to compare the two Laplacians as in Lemma 3.2. The reason why we chose not to do this is because in our main theorems we do not want any assumption on the Levi-Civita connection, but only on the canonical connection.

\section{The Schwarz lemma}

In this section, we prove Theorems 1.1 and 1.2. Using Cartan's formalism of moving frames, and the canonical connection, we prove in (5.9) a generalization of a formula due to Chern and $\mathrm{Lu}$ [18] in the integrable case. The Schwarz lemma then follows at once from the maximum principle, Theorem 4.1. The corresponding formula for the volume form is much easier, and already appears in [11].

Let $(M, J, g)$ and $(\tilde{M}, \tilde{J}, \tilde{g})$ be two almost-Hermitian manifolds of dimensions $2 n$ and $2 \tilde{n}$, respectively, and let $f: M \rightarrow \tilde{M}$ be an almost-complex mapping. Let $\left\{e_{i}\right\}$ and $\left\{\theta^{i}\right\}$ be local unitary frames and coframes for $g$ on $M$ and let $\left\{\tilde{e}_{i}\right\}$ and $\left\{\tilde{\theta}^{i}\right\}$ be those for $\tilde{g}$ on $\tilde{M}$. Let $\nabla$ and $\tilde{\nabla}$ be the canonical connections for $(M, J, g)$ and $(\tilde{M}, \tilde{J}, \tilde{g})$, respectively. We will use $\theta_{j}^{i}, \Theta^{i}, \Omega_{j}^{i}$ and $\tilde{\theta}_{\beta}^{\alpha}, \tilde{\Theta}^{\alpha}, \tilde{\Omega}_{\beta}^{\alpha}$ to denote the connection 1-forms, torsion and curvature for $\nabla$ and $\tilde{\nabla}$, respectively. Here we use roman letters $i, j, k, \ldots=1,2, \ldots, n$ for indices on $M$ and Greek letters $\alpha, \beta, \ldots=1,2, \ldots, \tilde{n}$ for indices on $\tilde{M}$. 
Since $f$ is almost-complex, there exist functions $a_{i}^{\alpha}$ on $M$ such that

$$
f^{*} \tilde{\theta}^{\alpha}=a_{i}^{\alpha} \theta^{i} .
$$

Define a function $u$ by $u=\operatorname{tr}_{g}\left(f^{*} \tilde{g}\right)$. Locally we can write $u$ as

$$
u=a_{i}^{\alpha} \overline{a_{i}^{\alpha}} .
$$

From now on, we will often omit writing the pullback $f^{*}$. Differentiating (5.1) and using the first structure equations for $\nabla$ and $\tilde{\nabla}$ we obtain

$$
\begin{aligned}
d \tilde{\theta}^{\alpha} & =d a_{i}^{\alpha} \wedge \theta^{i}-a_{i}^{\alpha} \theta_{j}^{i} \wedge \theta^{j}+a_{i}^{\alpha} \Theta^{i} \\
& =-a_{i}^{\beta} \tilde{\theta}_{\beta}^{\alpha} \wedge \theta^{i}+\tilde{\Theta}^{\alpha} .
\end{aligned}
$$

Rearranging this gives

$$
\left(d a_{i}^{\alpha}+\tilde{\theta}_{\beta}^{\alpha} a_{i}^{\beta}-a_{j}^{\alpha} \theta_{i}^{j}\right) \wedge \theta^{i}=\tilde{\Theta}^{\alpha}-a_{i}^{\alpha} \Theta^{i} .
$$

Since the right-hand side has no $(1,1)$ component, it follows that we can define functions $a_{i k}^{\alpha}$ by

$$
d a_{i}^{\alpha}+\tilde{\theta}_{\beta}^{\alpha} a_{i}^{\beta}-a_{j}^{\alpha} \theta_{i}^{j}=a_{i k}^{\alpha} \theta^{k} .
$$

Now apply the exterior derivative to both sides of this equation, substitute from the structure equations and (5.4), and cancel some terms to obtain

$$
a_{i}^{\beta} \tilde{\Omega}_{\beta}^{\alpha}+a_{i k}^{\beta} \theta^{k} \wedge \tilde{\theta}_{\beta}^{\alpha}-a_{j}^{\alpha} \Omega_{i}^{j}-a_{j k}^{\alpha} \theta^{k} \wedge \theta_{i}^{j}=d a_{i k}^{\alpha} \wedge \theta^{k}+a_{i k}^{\alpha}\left(-\theta_{j}^{k} \wedge \theta^{j}+\Theta^{k}\right),
$$

which can be rewritten as

$$
\left(d a_{i k}^{\alpha}-a_{i j}^{\alpha} \theta_{k}^{j}+a_{i k}^{\beta} \tilde{\theta}_{\beta}^{\alpha}-a_{j k}^{\alpha} \theta_{i}^{j}\right) \wedge \theta^{k}=a_{i}^{\beta} \tilde{\Omega}_{\beta}^{\alpha}-a_{j}^{\alpha} \Omega_{i}^{j}-a_{i k}^{\alpha} \Theta^{k} .
$$

Define functions $a_{i k \ell}^{\alpha}$ and $a_{i k \bar{\ell}}^{\alpha}$ by

$$
d a_{i k}^{\alpha}-a_{i j}^{\alpha} \theta_{k}^{j}+a_{i k}^{\beta} \tilde{\theta}_{\beta}^{\alpha}-a_{j k}^{\alpha} \theta_{i}^{j}=a_{i k \ell}^{\alpha} \theta^{\ell}+a_{i k \ell}^{\alpha} \overline{\theta^{\ell}} .
$$

Then taking the $(1,1)$ part of $(5.5)$ we obtain

$$
a_{i k \bar{\ell}^{\alpha}}^{\alpha} \wedge \overline{\theta^{\ell}}=-a_{i}^{\beta} \tilde{R}_{\beta \gamma \bar{\delta}}^{\alpha} \tilde{\theta}^{\gamma} \wedge \overline{\tilde{\theta}^{\delta}}+a_{j}^{\alpha} R_{i k \bar{\ell}}^{j} \theta^{k} \wedge \overline{\theta^{\ell}} .
$$

We now wish to calculate $d u$. Using (5.4) we have

$$
d u=\overline{a_{i}^{\alpha}} a_{i k}^{\alpha} \theta^{k}+a_{i}^{\alpha} \overline{a_{i k}^{\alpha} \theta^{k}},
$$


which means $\partial u=\overline{a_{i}^{\alpha}} a_{i k}^{\alpha} \theta^{k}, \bar{\partial} u=a_{i}^{\alpha} \overline{a_{i k}^{\alpha} \theta^{k}}$. Then

$$
\begin{aligned}
d \partial u & =a_{i k}^{\alpha} d \overline{a_{i}^{\alpha}} \wedge \theta^{k}+\overline{a_{i}^{\alpha}} d a_{i k}^{\alpha} \wedge \theta^{k}+\overline{a_{i}^{\alpha}} a_{i k}^{\alpha} d \theta^{k} \\
& =a_{i k}^{\alpha} \overline{a_{i \ell}^{\alpha} \theta^{\ell}} \wedge \theta^{k}+\overline{a_{i}^{\alpha}}\left(a_{i k \ell}^{\alpha} \theta^{\ell}+a_{i k \bar{\ell}}^{\alpha} \overline{\theta^{\ell}}\right) \wedge \theta^{k}+\overline{a_{i}^{\alpha}} a_{i k}^{\alpha} \Theta^{k}
\end{aligned}
$$

where we have used (5.4), (5.6) and the first structure equation. Hence

$$
(d \partial u)^{(1,1)}=-a_{i k}^{\alpha} \overline{a_{i \ell}^{\alpha}} \theta^{k} \wedge \overline{\theta^{\ell}}-\overline{a_{i}^{\alpha}} a_{i k \bar{\ell}}^{\alpha} \theta^{k} \wedge \overline{\theta^{\ell}} .
$$

Substituting from (5.7) we have

$$
(d \partial u)^{(1,1)}=\left(-a_{i k}^{\alpha} \overline{a_{i \ell}^{\alpha}}+\overline{a_{i}^{\alpha}} a_{i}^{\beta} \tilde{R}_{\beta \gamma \delta}^{\alpha} a_{k}^{\gamma} \overline{a_{\ell}^{\delta}}-\overline{a_{i}^{\alpha}} a_{j}^{\alpha} R_{i k \bar{\ell}}^{j}\right) \theta^{k} \wedge \overline{\theta^{\ell}}
$$

Then from Lemma 3.1 we obtain

$$
\frac{1}{2} \Delta u=\left|a_{i k}^{\alpha}\right|^{2}-\overline{a_{i}^{\alpha}} a_{i}^{\beta} a_{k}^{\gamma} \overline{a_{k}^{\delta}} \tilde{R}_{\beta \gamma \delta}^{\alpha}+\overline{a_{i}^{\alpha}} a_{j}^{\alpha} R_{i \bar{j}}^{\prime} .
$$

If the second Ricci curvature of $g$ is bounded below by $-K_{1}$ and the bisectional curvature of $\tilde{g}$ is bounded above by $-K_{2}<0$, then we obtain

$$
\frac{1}{2} \Delta u \geq K_{2} u^{2}-K_{1} u
$$

Then Theorem 4.1 gives that

$$
\operatorname{tr}_{g} f^{*} \tilde{g}=u \leq \frac{K_{1}}{K_{2}}
$$

which proves Theorem 1.1 since $f^{*} \tilde{g} \leq u g$.

Now assume that $M$ and $\tilde{M}$ have the same dimension $2 n$. Define a function

$$
v=\frac{\operatorname{det} f^{*} \tilde{g}}{\operatorname{det} g},
$$

so that $f^{*} d V_{\tilde{g}}=v d V_{g}$. Then $f$ is non-degenerate precisely when $v>0$ and is totally degenerate when $v \equiv 0$. Locally $v=|\nu|^{2}$ where $\nu=\operatorname{det}\left(a_{i}^{\alpha}\right)$. A computation in Section 3 of [11] (see also [24, Lemma 3.2]) gives

$$
\frac{1}{2} \Delta v=v R-v \tilde{R}_{\alpha \bar{\beta}} a_{i}^{\alpha} \overline{a_{i}^{\beta}}
$$

So if the scalar curvature of $g$ is bounded below by $-n K_{1}$ and the first Ricci curvature of $\tilde{g}$ is bounded above by $-K_{2}$, with $K_{2}>0$, then we get

$$
\frac{1}{2} \Delta v \geq K_{2} u v-n K_{1} v \geq n K_{2} v^{1+1 / n}-n K_{1} v,
$$


where we used the arithmetic-geometric mean inequality. Then Theorem 4.1 gives that

$$
v \leq\left(\frac{K_{1}}{K_{2}}\right)^{n}
$$

which proves Theorem 1.2.

\section{Product of almost-complex manifolds}

In this section we prove Theorem 1.3. We adapt the argument in [23] to our case, using again local holomorphic discs instead of complex coordinates, and applying our Theorem 1.1 and Proposition 4.1.

Suppose $M=X \times Y$ is the product of two almost-complex manifolds of (real) dimensions $2 n$ and $2 m$, respectively. Assume for a contradiction that $M$ admits a complete almost-Hermitian metric $g$ with torsion and $(2,0)$ part of the curvature bounded, and with bisectional curvature bounded between two negative constants such that

$$
-C_{1}<B(V, W)<-C_{2}<0
$$

holds for all $V, W \in T^{\prime} M$. Fix a point $q \in Y$ and choose $F: D \rightarrow Y$ a $J$-holomorphic disc with $F(0)=q$ and $F_{*}(e) \neq 0$. Here again $D \subset \mathbb{C}$ is the unit disc and the existence of such a map is given by [13]. Moreover, up to shrinking the disc, we can assume that the $F$ is an immersion, so that the vector field $V=F_{*}(\partial / \partial z) \in T^{\prime} Y$ does not vanish on the image of $F$, and that $T^{\prime} Y$ can be trivialized in a neighborhood of the image. For each $x \in X$ define a map $G_{x}: D \rightarrow M$ by sending $z$ to $(x, F(z))$. Each $G_{x}$ is almostcomplex with respect to the given almost-complex structures and moreover the map $G: X \times D \rightarrow M$ given by $G(x, z)=G_{x}(z)$ is also almost-complex. Take $\eta \in C_{c}^{\infty}(D)$ to be a smooth non-trivial cutoff function, with $0 \leq \eta \leq 1$, and define a smooth positive function $f$ on $M$ by

$$
f(x, y)=f(x)=\int_{D} \eta G_{x}^{*} g .
$$

Equip $D$ with the Poincaré metric $g_{0}$, and apply Theorem 1.1 to $G_{x}$ to get

$$
G_{x}^{*} g \leq \frac{1}{C_{2}} g_{0}
$$

which implies that $f$ is bounded above. Now fix a point $p=\left(x_{0}, q\right) \in M$ and pick $\left\{e_{1}, \ldots, e_{n}\right\}$ a local frame on $X$ around $x_{0}$, and $\left\{e_{n+1}, \ldots, e_{n+m}\right\}$ 
a local frame on $Y$ around the image of $F$. Then, by abusing notation, we denote by $\left\{e_{1}, \ldots, e_{n+m}\right\}$ the induced frame on $M$, which in general is not unitary. Then locally the Hermitian metric $g$ on $T^{\prime} M$ can be written as $g_{i j} \theta^{i} \otimes \overline{\theta^{j}}$, and on the image of $G_{x}, V$ is of the form $V=V^{j} e_{j}$, where $n+1 \leq j \leq n+m$. Moreover we can assume that at $p$ we have $g_{i \bar{j}}=\delta_{i j}$ for $1 \leq i, j \leq n$. Then we can write

$$
f=\sqrt{-1} \int_{D} \eta g_{j \bar{k}} V^{j} \overline{V^{k}} d z \wedge d \bar{z} .
$$

Since $f$ is constant along $Y$, we see that $f_{j}=f_{\bar{j}}=0$ for $n+1 \leq j \leq n+m$. From now on fix $1 \leq i \leq n$, and notice that on $X \times D$ we have, by abusing notation, $\left[\overline{e_{i}}, \partial / \partial z\right]=0$. Hence

$$
0=G_{*}\left(\left[\overline{e_{i}}, \partial / \partial z\right]\right)=\left[\overline{e_{i}}, V\right]
$$

and so $(2.3)$ gives $0=\theta^{j}\left(\left[\overline{e_{i}}, V\right]\right)=V_{\bar{i}}^{j}$ for all $n+1 \leq j \leq n+m$. Hence

$$
\begin{aligned}
f_{i} & =\sqrt{-1} \int_{D} \eta g_{j \bar{k}} V_{i}^{j} \overline{V^{k}} d z \wedge d \bar{z} \\
f_{i \bar{i}} & =\sqrt{-1} \int_{D} \eta g_{j \bar{k}}\left(V_{i \bar{i}}^{j} \overline{V^{k}}+V_{i}^{j} \overline{V_{i}^{k}}\right) d z \wedge d \bar{z} \\
& \geq \sqrt{-1} \int_{D} \eta g_{j \bar{k}} V_{i \bar{i}}^{j} \overline{V^{k}} d z \wedge d \bar{z}
\end{aligned}
$$

where we have used that $\nabla g=0$. Now proceeding as in the derivation of (4.7), we obtain

$$
\begin{aligned}
g_{j \bar{k}} V_{i \bar{i}}^{j} \overline{V^{k}} & =g_{j \bar{k}}\left(V_{\bar{i} \bar{i}}^{j} \overline{V^{k}}-V^{\ell} \overline{V^{k}} R_{\ell i \bar{i}}^{j}\right)=-g_{j \bar{k}} V^{\ell} \overline{V^{k}} R_{\ell i \bar{i}}^{j} \\
& \geq C_{2} g_{j \bar{k}} V^{j} \overline{V^{k}} g_{i \bar{i}} .
\end{aligned}
$$

Denote by $h$ the almost-Hermitian metric on $X$ obtained by restricting $g$ to $X \times\{q\}$. In [14] it is proved that the bisectional curvature of an almostcomplex submanifold is always less than the one of the ambient space, and so the bisectional curvature of $h$ is bounded above by $-C_{2}$. The projection $\pi_{1}:(M, g) \rightarrow(X, h)$ is almost-complex and Theorem 1.1 gives

$$
\pi_{1}^{*} h \leq C_{3} g
$$

where $C_{3}=(n+m) C_{1} / C_{2}$. This implies that

$$
g_{i \bar{i}}(x, q) \leq C_{3} g_{i \bar{i}}(x, y)
$$


for any $(x, y)$ near $p$. Combining $(6.1),(6.2)$ and (6.3) we obtain

$$
f_{i \bar{i}}(x, y) \geq \frac{C_{2}}{C_{3}} g_{i \bar{i}}(x, q) f(x, y)
$$

and so at $p$ we obtain $f_{i \bar{i}} \geq \alpha f$, where $\alpha=\frac{C_{2}}{C_{3}}>0$. Summing up and using Lemma 3.1 we obtain $\frac{1}{2} \Delta f \geq n \alpha f$, and Proposition 4.1 applied to $-f$ gives $f=0$, which is absurd.

\section{Acknowledgments}

First of all I would like to thank my thesis advisor Professor S.-T. Yau for suggesting this problem, sharing his ideas with me and for his constant support. I would also like to thank Ben Weinkove for many useful discussions and for reading an earlier version of this paper, and Harish Seshadri for suggesting this generalization of his results.

\section{References}

[1] L. Ahlfors, An extension of Schwarz's lemma, Trans. Amer. Math. Soc. 43(3) (1938), 359-364.

[2] E. Calabi, An extension of E. Hopf's maximum principle with an application to Riemannian geometry, Duke Math. J. 25 (1958), 45-56.

[3] Z. Chen, Complex geometry in China, Several complex variables in China, Contemp. Math., 142, American Mathematical Society., Providence, RI, 1993, 1-14.

[4] Z. Chen and H. Yang, Estimation of the upper bound on the Levi form of the distance function on Hermitian manifolds and some of its applications (Chinese), Acta Math. Sinica 27(5) (1984), 631-643.

[5] S.-S. Chern, On holomorphic mappings of hermitian manifolds of the same dimension, Entire functions and related parts of analysis (Proc. Sympos. Pure Math., La Jolla, California, 1966), American Mathematical Society, Providence, RI, 1968, 157-170.

[6] B. Coupet, H. Gaussier, and A. Sukhov, Some aspects of analysis on almost complex manifolds with boundary, Preprint, 2007, arXiv:math/0701576.

[7] S.K. Donaldson, Two-forms on four-manifolds and elliptic equations, Inspired by S.S. Chern, World Scientific, 2006. 
[8] J. Eells and L. Lemaire, A report on harmonic maps, Bull. London Math. Soc. 10(1) (1978), 1-68.

[9] P. Gauduchon, Hermitian connections and Dirac operators, Boll. Unione Mat. Ital. B 11(2) (1997), suppl, 257-288.

[10] S.I. Goldberg, and Z. Har'El, A general Schwarz lemma for Riemannian-manifolds, Bull. Soc. Math. Grèce (NS) 18(1) (1977), 141-148.

[11] S.I. Goldberg and Z. Har'El, Mappings of almost Hermitian manifolds, J. Differential Geom. 14(1) (1979), 67-80.

[12] A. Gray, Nearly Kähler manifolds, J. Differential Geom. 4 (1970), 283-309.

[13] S. Ivashkovich and J.-P. Rosay, Schwarz-type lemmas for solutions of $\bar{\partial}$-inequalities and complete hyperbolicity of almost complex manifolds, Ann. Inst. Fourier (Grenoble) 54(7) (2004), 2387-2435.

[14] S. Kobayashi, Almost complex manifolds and hyperbolicity, Results Math. 40(1-4) (2001), 246-256.

[15] S. Kobayashi and K. Nomizu, Foundations of differential geometry, Vol I, Interscience Publishers, John Wiley \& Sons, New York-London, 1963.

[16] A. Lichnerowicz, Applications harmoniques et variétés kähleriennes (French), Symposia mathematica, Vol. III (INDAM, Rome, 1968/1969) Academic Press, London, 341-402.

[17] K. Liu, X. Sun, and S.-T. Yau, Canonical metrics on the moduli space of Riemann surfaces I, J. Differential Geom. 68(3) (2004), 571-637.

[18] Y. Lu, Holomorphic mappings of complex manifolds, J. Differential Geom. 2 (1968), 299-312.

[19] A. Nijenhuis and W.B. Woolf, Some integration problems in almostcomplex and complex manifolds, Ann. of Math. 77 (1963), 424-489.

[20] H.L. Royden, The Ahlfors-Schwarz lemma in several complex variables, Comment. Math. Helv. 55(4) (1980), 547-558.

[21] R. Schoen and S.-T. Yau, Lectures on differential geometry, International Press, Cambridge, MA, 1994.

[22] H. Seshadri, Negative sectional curvature and the product complex structure, Math. Res. Lett. 13(2-3) (2006), 495-500. 
[23] H. Seshadri and F. Zheng, Complex product manifolds cannot be negatively curved, Preprint, 2008, arXiv:math/0801.0284, to appear in Asian J. Math.

[24] V. Tosatti, B. Weinkove, and S.-T. Yau, Taming symplectic forms and the Calabi-Yau equation, Proc. London. Math. Soc. 2008; doi: $10.1112 / \mathrm{plms} / \mathrm{pdn} 008$.

[25] B. Weinkove, The Calabi-Yau equation on almost-Kähler fourmanifolds, J. Differential Geom. 76(2) (2007), 317-349.

[26] P.C. Yang, On Kähler manifolds with negative holomorphic bisectional curvature, Duke Math. J. 43(4), (1976), 871-874.

[27] S.-T. Yau, Harmonic functions on complete Riemannian manifolds, Comm. Pure Appl. Math. 28 (1975), 201-228.

[28] S.-T. Yau, A general Schwarz lemma for Kähler manifolds, Amer. J. Math. 100(1) (1978), 197-203.

[29] F. Zheng, Non-positively curved Kähler metrics on product manifolds, Ann. of Math. 137(3) (1993), 671-673.

Department of Mathematics

HARVARD UNIVERSITY

CAMBridge, MA 02138

USA

E-mail address: tosatti@math.harvard.edu

RECEIVED July 12, 2007 